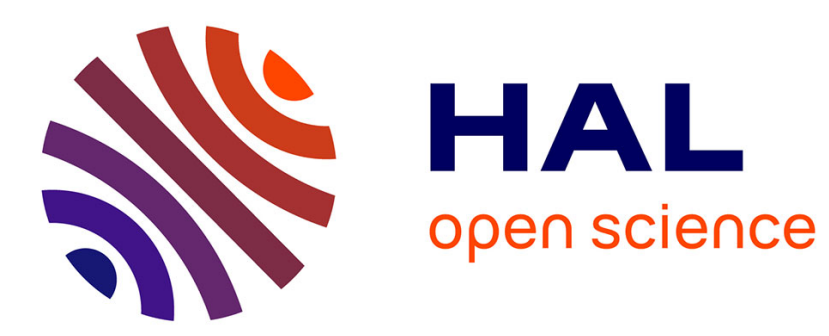

\title{
FLASHfrom accelerator test facility to the first single-pass soft x-ray free-electron laser
}

Jochen R Schneider

\section{To cite this version:}

Jochen R Schneider. FLASHfrom accelerator test facility to the first single-pass soft x-ray freeelectron laser. Journal of Physics B: Atomic, Molecular and Optical Physics, 2010, 43 (19), pp.194001. 10.1088/0953-4075/43/19/194001 . hal-00569834

\section{HAL Id: hal-00569834 \\ https://hal.science/hal-00569834}

Submitted on 25 Feb 2011

HAL is a multi-disciplinary open access archive for the deposit and dissemination of scientific research documents, whether they are published or not. The documents may come from teaching and research institutions in France or abroad, or from public or private research centers.
L'archive ouverte pluridisciplinaire HAL, est destinée au dépôt et à la diffusion de documents scientifiques de niveau recherche, publiés ou non, émanant des établissements d'enseignement et de recherche français ou étrangers, des laboratoires publics ou privés. 


\title{
FLASH - From Accelerator Test Facility to the First Single-Pass Soft X-ray FEL
}

\author{
Jochen R Schneider \\ Deutsches Elektronen-Synchrotron DESY, Center for Free-Electron Laser Science \\ CFEL, Notkestrasse 85, 22603 Hamburg, Germany \\ E-mail: Jochen.Schneider@DESY.DE
}

\begin{abstract}
The development from a test facility for the TESLA project of a Linear Collider with Integrated X-ray Free-Electron Laser to the world's first soft X-ray FEL user facility is described. In the wavelength range from 6.5 to $60 \mathrm{~nm}$ FLASH provides short pulses ( $25 \mathrm{fsec}$ ) containing $\sim 10^{12}$ photons in laterally coherent beams with brilliance about 9 orders of magnitudes higher than achieved at the best synchrotron radiation storage ring facilities today. FLASH opened a new area in photon science where matter in non equilibrium states is studied with atomic resolution in space and time.
\end{abstract}

PACS numbers: $41.60 \mathrm{Cr}, 29.20 . \mathrm{Ej}$

\section{Introduction}

FLASH, the Free-Electron Laser in Hamburg, was the first FEL user facility in the spectral range of the VUV-XUV. It was initiated as a test facility for the project of the "TESLA Linear Collider with Integrated X-ray Free-Electron Laser Facility". It is fair to say, without the great engagement of the particle physics community for the linear collider project, FLASH at DESY would not exist today. In the 1990ies the photon science community at large was still rather reserved concerning FEL facilities. On the other hand a number of groups accepted the challenge and pioneered a new area for photon science with building FLASH and doing experiments at FLASH. For the first time it is now possible to study matter, including matter in extreme conditions and in non-equilibrium states, with atomic resolution in space and time. The often used buzzwords are "take movies instead of pictures". This start in a new area of science is documented by the collection of articles on science with FLASH collected in this special issue of J. Phys. B.

The paper is organized as follows: After sketching the origin of the concepts for single-pass $\mathrm{X}$-ray free-electron lasers and first steps to develop the science case, the development at DESY in Hamburg, which lead to the construction of the TESLA Test Facility TTF1, is described in some detail. After presentation of layout and performance of this test facility the first experiments on clusters are described. Next the layout and performance of the second stage of expansion of the facility (see figure 1), first called TTF2 and later renamed to FLASH, are presented followed by a description of the first single-shot coherent imaging experiment performed at a FEL. The summary starts with a sketch of the upgrades of FLASH, under way or planed, followed by a short presentation of the other FELs in operation or under construction, projects in an advanced planning stage or currently discussed. Some observations concerning usage of FEL facilities conclude the paper. Beamlines, diagnostics and instrumentation at FLASH are discussed in the following paper by $\mathrm{J}$. Feldhaus. 


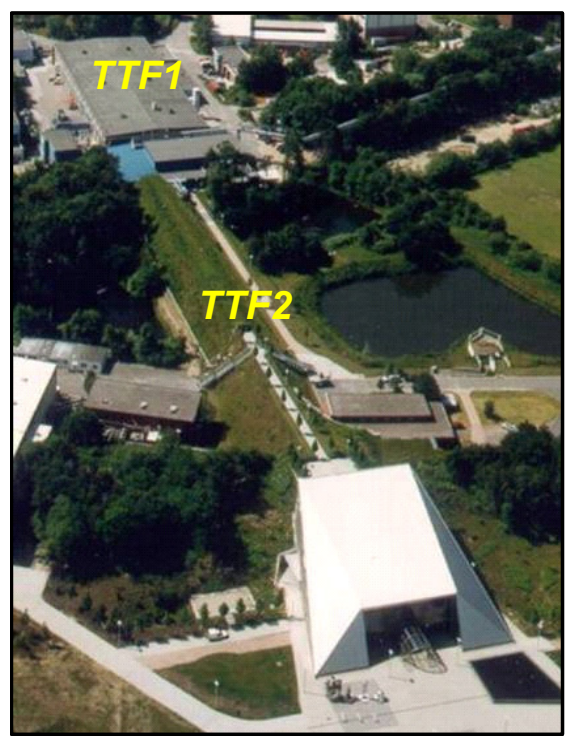

Figure 1. The free-electron laser facility at DESY.

The TESLA Test Facility TTF1 was the start configuration with focus on development and test of the superconducting linac for the project of a Linear Collider for Particle Physics with Integrated X-ray Free-Electron Laser Facility. From 2003 on TTF1 was expanded to TTF2, an FEL user facility for the spectral range of soft X-rays including a new tunnel and a new experimental hall (in the foreground). In April 2006 the facility was renamed to FLASH: Free-Electron Laser in Hamburg

\section{Start at DESY - The TESLA Collaboration}

The theoretical basis for X-ray free-electron lasers has been established in the 1980s by two papers by A.M. Kondratenko and E.L. Saldin [1], and R. Bonifacio, C. Pellegrini and L.M. Narducci [2]. They stimulated a lively discussion in the accelerator community in a series of workshops organized by Brookhaven National Laboratory and the Stanford Linear Accelerator Laboratory SLAC in the early 1990s. Already in 1992 detailed suggestions were made to use the existing SLAC linac equipped with low emittance guns to drive $4 \mathrm{~nm}$ to $0.1 \mathrm{~nm}$ FELs.

At that time DESY shared the vision of a large part of the particle physics community to build a TeV electron-positron linear collider and pursued this project vigorously when Björn $\mathrm{H}$. Wiik became Director General of the laboratory. However, there were clear indications from the funding agencies in Germany that such a large research facility should be attractive not just for the particle physics community and DESY developed within an international collaboration a Conceptual Design for a $500 \mathrm{GeV}^{+}-\mathrm{e}^{-}$Linear Collider with Integrated X-ray Laser Facility[3].

Based on the good experience with superconducting technology at the large hadron-lepton collider HERA at DESY and the need for high luminosity at the linear collider, the challenge was accepted to realize the accelerator in superconducting RF technology in a large international effort. The so called TESLA collaboration was founded which by the end of 2002 included 50 institutions out of 12 countries. The ambitious goal was to increase the gradient of the accelerator by a factor of 5 and to reduce the cost for the cryomodules by a factor of 5 , which today is achieved.

In March 2001 the TESLA collaboration presented the Technical Design Report on a Linear Collider with an Integrated X-ray Free-Electron Laser Facility at a colloquium at DESY on Scientific Perspectives and Technical Realization of TESLA which attracted more than 1100 scientists, $40 \%$ from abroad. The reaction of the science community at large and the funding agencies was positive. After an evaluation of the TESLA proposal by the German Science Council the German Government decided in February 2003 that the XFEL part of the proposal should be realized in Hamburg as a European project with Germany paying half of the cost. Construction of the European XFEL started in January 2009 and first electron beams are expected for 2014. 
In the 1990s the interest of the photon science community was rather focused on hard X-ray FELs. For example, in the US in 1999 a panel on novel light sources initiated by the Department of Energy and chaired by S. Leone concluded that the most exciting potential advance in the area of innovative science is most likely in the hard X-ray region, in the range of $8-20 \mathrm{keV}$, and even higher. Today, encouraged by the scientific results obtained at the first FEL user facility FLASH at DESY, the potential of soft X-ray FELs is intensely discussed in the community and a number of new projects have been presented.

As part of the R\&D for the TESLA project DESY needed to build a test facility for the development of the superconducting linear accelerator and it was decided to combine this facility with a free-electron laser in the spectral range of the VUV. It was easy to formulate a convincing scientific case, the Conceptual Design Report for the FEL was submitted in June 1995, the project was approved in October of the same year, and construction of the so called TESLA Test Facility started.

\section{TESLA Test Facility (TTF1)}

TTF1, the TESLA Test Facility in its first stage of expansion, consisted of a low emittance laser driven photocathode electron gun, a pre-accelerator, two superconducting accelerator modules, three sections of undulator structures each $4.5 \mathrm{~m}$ long and various electron and photon beam diagnostics (see figure 2). In addition a first experiment to study clusters was installed.

In principle superconducting linear accelerators provide the possibility of $\mathrm{CW}$ operation. However, as shown in figure 3, at DESY a pulse train structure was chosen as the optimal bunch time pattern for the project of a Linear Collider with Integrated Free-Electron Laser. The injector is based on a laser driven $\mathrm{Cs}_{2} \mathrm{Te}$ photocathode which is installed in a 1.6-cell normal-conducting RF cavity operated at $1.3 \mathrm{GHz}$. The bunch compressor provides the high peak currents needed for lasing. The undulator is a permanent magnet device with a fixed $12 \mathrm{~mm}$ gap and a period of 2.73 $\mathrm{cm}$. The electron beam is dumped in an underground solid state dump. Photon beam diagnostics included a detector to determine the pulse energy and a spectrometer to measure the spectral distribution of the X-ray pulses shot by shot.

Lasing at saturation at a wavelength of $98 \mathrm{~nm}$ was first reached September 10, 2001. Aftermath tunability in the range from 80 to $120 \mathrm{~nm}$ was demonstrated, as well as a very high degree of lateral coherence of the beam. The duration of GW radiation pulses was estimated to be between 30 and 100 femtoseconds, a tool for direct measurement of such ultra-short pulses was not available at the time. For details and parameter lists see [4-5]. The peak brilliance of the FEL radiation is $\sim 10^{30}$ photons $/\left(\mathrm{s} \mathrm{mrad}^{2} \mathrm{~mm}^{2} 0.1 \% \mathrm{BW}\right)$ and thus about 9 orders of magnitude higher than available at the best synchrotron radiation storage rings. At the FEL one obtains about $10^{12}$ photons in a pulse of $\sim 25$ fsec duration, i.e. as many photons as we get today at the best electron storage rings per second.

All first generation FELs are based on the principle of Self-Amplified Spontaneous Emission (SASE). Here the electron density of the bunch is modulated by a resonant process taking place in the combined presence of the periodic transverse magnetic field of the undulator and the electromagnetic radiation. The wavelength of the modulation is equal to that of the radiation produced by the undulator, and finally to the wavelength of the FEL radiation. As a consequence the FEL wavelength can be tuned by either changing the magnetic gap of the undulator or the energy of the electron beam. The modulation process starts from shot noise leading to a series of coherently modulated sections in the electron bunch, which correspond to different longitudinal modes in the beam and to spikes in the spectral distribution as well as in the time structure of the FEL pulse. As shown in figure 4 the number of longitudinal modes depends on the length of the electron bunch, which implies that in principle one can achieve single mode FEL radiation in a SASE FEL if the electron bunch can be sufficiently compressed and still reaching the peak current needed for lasing. All properties of the FEL beam fluctuate and those important for the interpretation of the experiment have to be monitored on a shot by shot basis. 
As an approach to avoid the spiky structure of the pulses which is inherent to the SASE process a so called Self-Seeding Option has been proposed for the FEL at TTF2 [6]. Figure 5 shows the schematic setup (side view) of the Self-Seeding Option which should provide narrow-band FEL radiation down to about $6 \mathrm{~nm}$ wavelength. After the first undulator section, FEL radiation and electrons are separated. While the electrons travel through the bypass represented by the four blue dipole magnets in the upper part of figure 5 , the photons propagate across the grating monochromator beamline below in order to provide a narrow-bandwidth seed that is overlayed with the electrons at the entrance of the second undulator section. Simulations show that for the FLASH parameters one can expect a spectral distribution of a single pulse instead of the ensemble of narrow spikes typical for a SASE FEL. The expected gain in peak as well as in average brilliance is about 2 orders of magnitude. The pulse duration is increased to $300 \mathrm{fsec}$, on the other hand, this mode of operation opens very interesting spectroscopic applications where high energy resolution is needed and longer pulse duration is acceptable.

For more information we refer to http://hasyweb.desy.de/flash_booklet/flash brochure.pdf, a brochure which describes the FLASH accelerator and the process of producing FEL radiation, as well as the beamlines, instruments and the experimental hall.

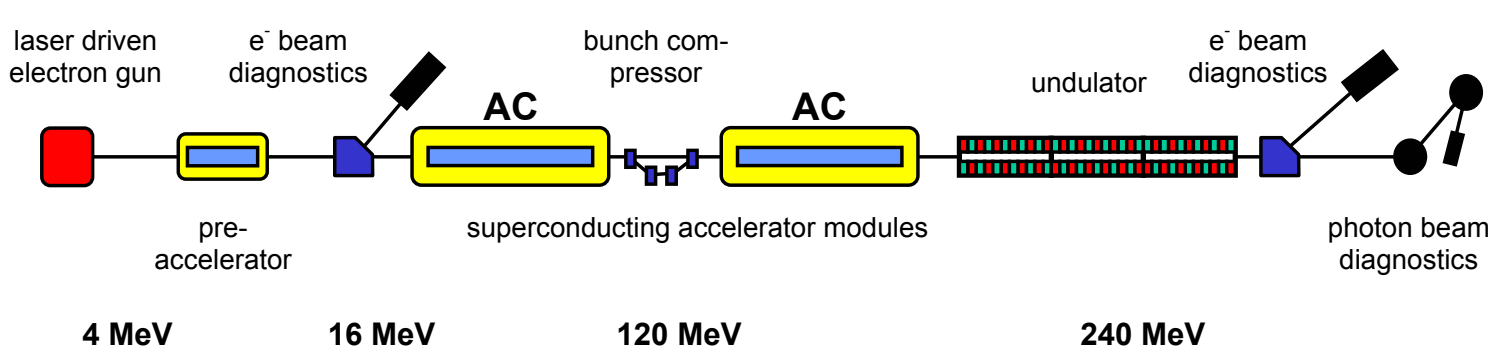

Figure 2. Schematic layout of the first stage of expansion of the TESLA Test Facility, called TTF1. The total length is approximately $120 \mathrm{~m}$, the electron energy $240 \mathrm{MeV}$.

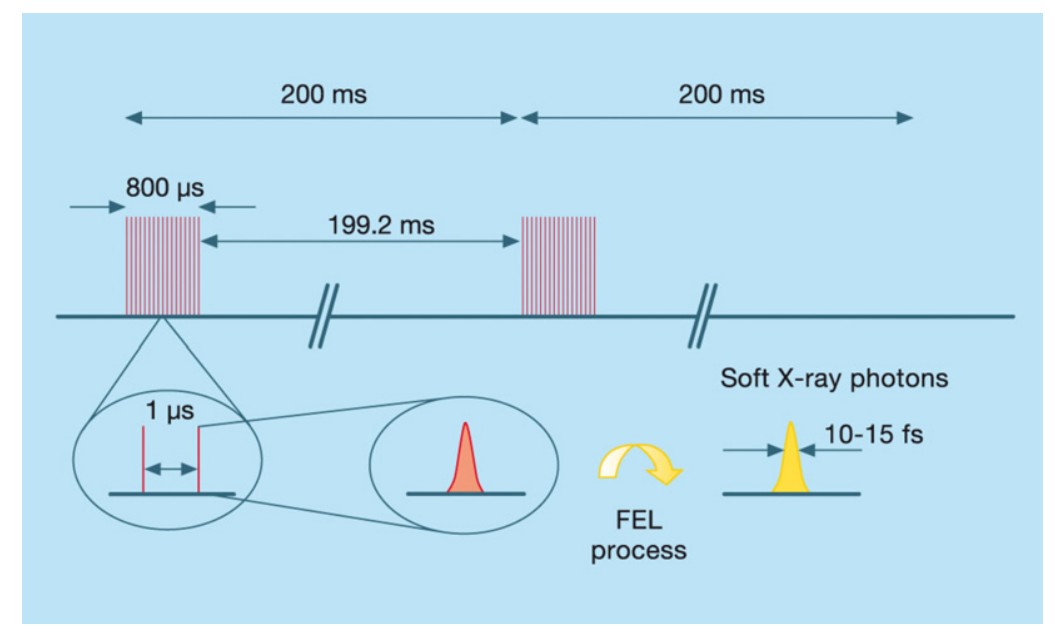

Figure 3. Electron bunch time pattern of TTF with $5 \mathrm{~Hz}$ repetition rate and up to 800 bunches in a $800-\mu$ s-long bunch train, i.e. up to 4000 photon flashes per second could be obtained. 


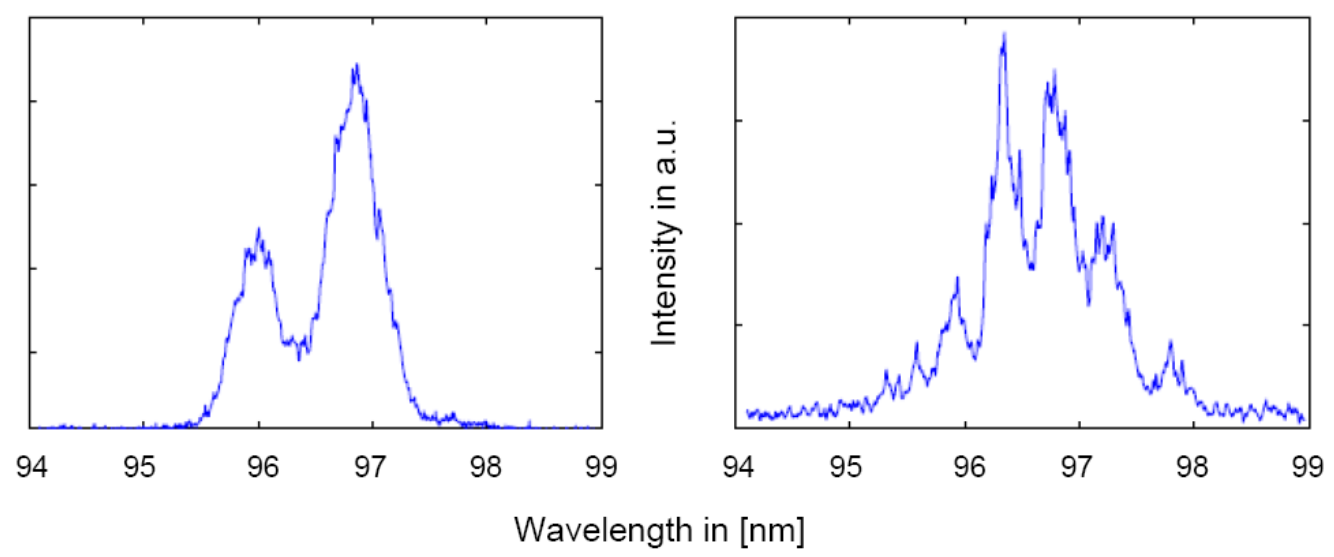

Figure 4. Energy spectra from short (duration $\sim 40 \mathrm{fsec}$ ) and long (duration $\sim 100$ fsec) FEL pulses at a wavelength of $98 \mathrm{~nm}$. The number of "spikes" corresponds to the number of longitudinal modes in the radiation pulse and increases with increasing electron bunch length [5].

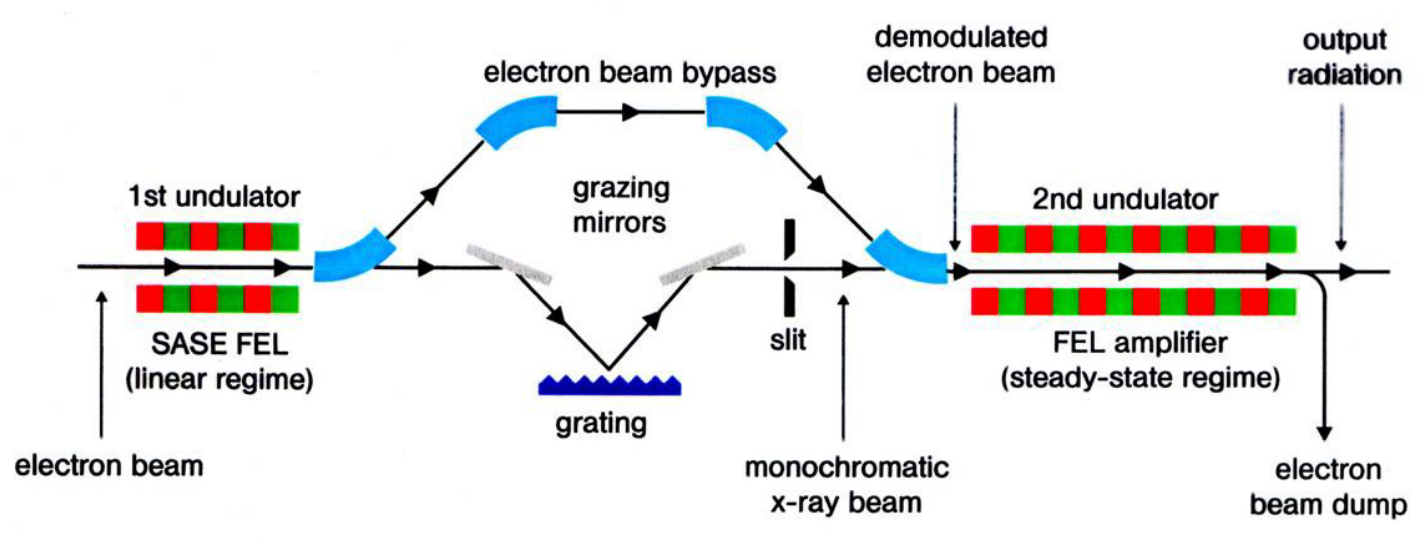

Figure 5. Schematic setup (side view) of the Self-Seeding Option for the FEL at TTF2, which can provide narrow-band FEL radiation down to about $6 \mathrm{~nm}$ wavelength [6].

\section{First FEL experiments on clusters}

For all experiments performed at free-electron lasers it is crucial to understand the interaction of the extremely intense, ultra-short X-ray pulses with matter on the atomic and molecular level. Therefore it was rather natural to perform the first experiments at the TESLA Test Facility on clusters which are intermediate between atoms and condensed matter. The group of T. Möller studied Xenon clusters using $98 \mathrm{~nm}$ FEL radiation at power densities up to $7 \times 10^{13} \mathrm{Wcm}^{-2}$ [7]. Figure 6 shows the principle layout of such experiments.

A pulsed beam of atoms or clusters was prepared by expanding Xe gas at high pressure through a small conical nozzle. The composition of the beam and the size of the clusters could be controlled by varying the gas pressure. The cluster beam passed through a small scimmer into the main chamber. The FEL beam was focused to a spot of $20 \mu \mathrm{m}$ in diameter by an elliptical mirror which could handle the extremely intense VUV beam very well. No deterioration of the beam quality was observed during the experiment. Off line investigations of the mirror properties performed later did not reveal any significant radiation damage. The pulse energy was measured with a calibrated channel-plate detector, which detects a well-defined percentage of FEL light scattered from a wire. The power density was calculated assuming pulse duration of $100 \mathrm{fsec}$. Figure 7 shows single shot time-of-flight mass spectra for Xe atoms and clusters. 
The most striking result is the big difference between the spectra measured at atoms and large clusters. Whereas only single charged ions are observed after irradiation of isolated atoms, atomic ions with charges up to $8+$ are observed when irradiating clusters, although the photon energy of $12.7 \mathrm{eV}$ is only slightly higher than the Xe ionization potential of $12.1 \mathrm{eV}$. As also shown in figure 7 this effect is strongly dependent on the power density of the photon pulses. At a power density of $7 \times 10^{13} \mathrm{Wcm}^{-2}$ each atom in large clusters absorbs up to $400 \mathrm{eV}$, corresponding to 30 photons during a time of about $100 \mathrm{fsec}$, the duration of the FEL pulse. Wabnitz et al. [7] suggest that the clusters are heated up very efficiently by the VUV radiation and electrons are emitted after acquiring sufficient energy before they finally disintegrate completely by Coulomb explosion. These very first results obtained at the SASE FEL of the TESLA Test Facility stimulated a broad discussion in the community and a wealth of further experimental and theoretical work. An overview of the field will be given by T. Möller in another contribution to this special issue of J. Phys. B.

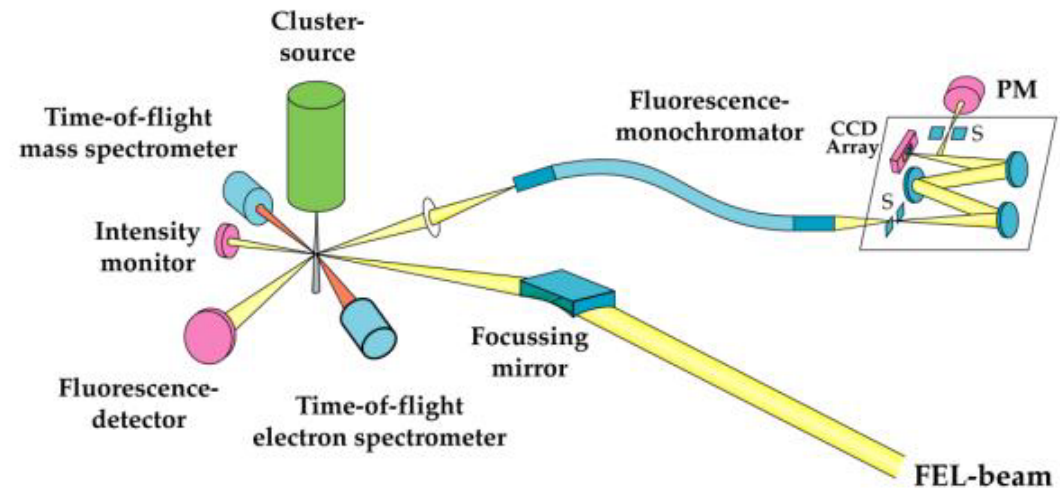

Figure 6. Layout of the cluster experiment.

The FEL beam ( $=98 \mathrm{~nm}$, bandwidth $\sim 0.5 \mathrm{~nm}$ ) is focused by a mirror to a spot size of about 20 $\mu \mathrm{m}$ in diameter where it interacts with a beam of clusters. The photon beam intensity and time-offlight mass- and/or electron spectra are measured on a shot by shot basis. In the first TTF experiments fluorescence radiation could not be measured.
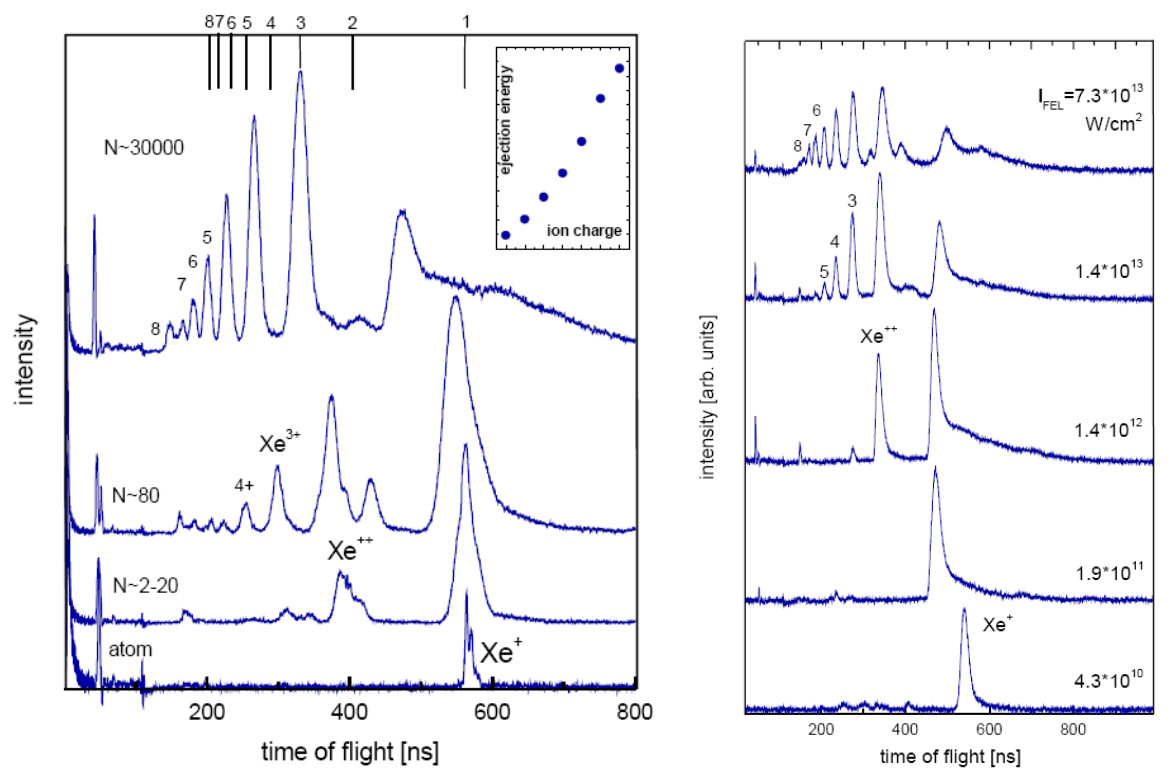

Figure 7. 
Time-of-flight mass spectra of ionization products of Xe atoms and clusters after irradiation with soft X-rays of $98 \mathrm{~nm}$ wavelength.

Left: Spectra recorded after ionization with pulses of an average power density of $2 \times 10^{13} \mathrm{Wcm}^{-2}$. The atomic spectrum (bottom trace) shows a splitting into several lines owing to the different isotopes. After irradiation of clusters, highly charged ions are detected and a strong dependence of the cluster size is observed. The mass peaks are rather broad and displaced with respect to the calculated flight times indicated by thin vertical lines (different charge states) in the uppermost part of the figure. This line shift indicates that the ions have high kinetic energies shown in the inset as a function of the charge for clusters containing $\mathrm{N}=1,500$ atoms.

Right: Spectra taken at clusters with $\mathrm{N}=1,500$ as a function of power density which was varied by either reducing the gain of the FEL or by moving the cluster beam out of the radiation focus. The intensity of highly charged ions increases with increasing power density [7].

\section{Layout and performance of TTF2/FLASH}

The operation of the first stage of expansion of the TESLA Test Facility, where emphasis was on development and test of the superconducting linac for the project of a Linear Collider for Particle Physics with Integrated X-ray Free-Electron Laser facility was concluded in December 2002. In the next phase, called TTF2, focus was on the construction of a soft X-ray free-electron laser user facility. Commissioning of the expanded facility started in fall 2004, first lasing at a wavelength of $32 \mathrm{~nm}$ was observed in January 2005. User operation officially started in August 2005.

The layout of TTF2 as in place in the fall of 2004 is shown in figure 8. Compared to the first stage of expansion of the TESLA Test Facility (figure 2) the most important changes are the increase of the electron energy from about 240 to $700 \mathrm{MeV}$ by adding/replacing cryomodules, the addition of a second bunch compressor, the installation of six undulators instead of three, a collimator section to protect the undulator against Bremsstrahlung and in case of electron beam steering accidents. The electron bypass allows accelerator studies without danger of damaging the undulators. Additionally an experimental station was installed in the bypass inside the tunnel for damage and other studies using the electron beam. The performance of the facility is described in ref [8]. Later a $6^{\text {th }}$ cryomodule was added and the electron energy increased to $\sim 1 \mathrm{GeV}$, which enabled lasing at $6.5 \mathrm{~nm}$, first observed in October 2007. Ref. [9] describes in detail the operation of the facility in the wavelength range from $13.7 \mathrm{~nm}$ in the fundamental to $4.6 \mathrm{~nm}$ in the $3^{\text {rd }}$ and to $2.75 \mathrm{~nm}$ in the $5^{\text {th }}$ harmonic.

In 2006/2007 the spectral range of FLASH was extended into the FIR by installing a longperiod undulator and a new IR beamline [10] (see figure 9). This way intense, truly synchronized IR pulses tunable in a broad spectral range are produced e.g. for two-color pump-probe experiments. The wavelength of the fundamental of the device can be tuned between $1 \mu \mathrm{m}$ and $200 \mu \mathrm{m}$ $(300 \mathrm{THz}$ to $1.5 \mathrm{THz}$ ). For wavelengths longer than $20 \mu \mathrm{m}$ pulse energies around $10 \mu \mathrm{J}$ are achieved. The first experiments at the beamline aimed at determining the spatial and temporal properties of individual pulses from FLASH and to unveil their temporal substructure [11].

The high peak current necessary for lasing cannot be produced in the electron gun, instead the electron bunches need to be compressed which is done in two steps. First an energy slope is imprinted on the bunch by acceleration on the falling slope of the RF field in the cavities. As a result the particles at the head of the bunch receive a smaller energy gain than those at the tail. Afterwards the particles pass through two magnetic chicanes where the electrons of larger energy travel shorter distance than the leading ones of smaller energy and thus are enabled to catch up. Due to a nonlinear effect in this process the final bunches do not possess the ideal narrow shape but consist of a leading spike of $\sim 50 \mathrm{fsec}$ duration and a long tail as shown in figure 10. The expected longitudinal electron distribution within the bunch consists of a $\sim 50$ fs long leading spike containing some $10 \%$ of the total charge with a peak current exceeding $1 \mathrm{kA}$. Lasing is possible only in this leading spike of the bunch. The duration of the FEL pulse was determined to $\sim 25 \pm 5 \mathrm{fsec}$ with a streak camera using the synchronized IR pulse of FLASH [11] and an interferometric approach [12]. 
Figure 11 shows the spectral peak brilliance calculated for FLASH at DESY, the Linac Coherent Light Source (LCLS) at SLAC in Stanford, and the European XFEL Facility in Hamburg in comparison with the performance of $3^{\text {rd }}$ generation synchrotron radiation facilities. Overall the gain with free-electron lasers amounts to approximately 9 orders of magnitude. It is interesting to note that the experimental results achieved at FLASH for the fundamental at $13.7 \mathrm{~nm}$ as well as for the $3^{\text {rd }}$ and $5^{\text {th }}$ harmonic very nicely reproduce the earlier theoretical predictions. With the higher harmonics FLASH reaches the water window. For an average pulse energy of $40 \mu \mathrm{J}$ of the fundamental at $13.7 \mathrm{~nm}$ a power of $0.25 \pm 0.1 \mu \mathrm{J}$ was measured for the $3^{\text {rd }}(4.6 \mathrm{~nm})$ and $10 \pm 4 \mathrm{~nJ}$ for the $5^{\text {th }}$ $(2.75 \mathrm{~nm})$ harmonic. In other words by going to higher order radiation the number of photons per pulse is reduced by two to four orders of magnitude. These weaker beams allow e.g. spectroscopic studies of condensed matter were the full beam intensity would create space charges masking the ground state properties of the investigated system. As an example, in combination with a synchronized optical laser, femtosecond time-resolved core-level photoelectron spectroscopy experiments were performed at FLASH using $118.5 \pm 0.2 \mathrm{eV}$ photons from the $3^{\text {rd }}$ harmonic [13].

To date all quantities measured at the VUV-XUV beams at the free-electron laser FLASH fit very well the theoretical predictions. Obviously the SASE process can be simulated well to great detail and as demonstrated recently at SLAC, this is also true for the spectral range of hard X-rays.

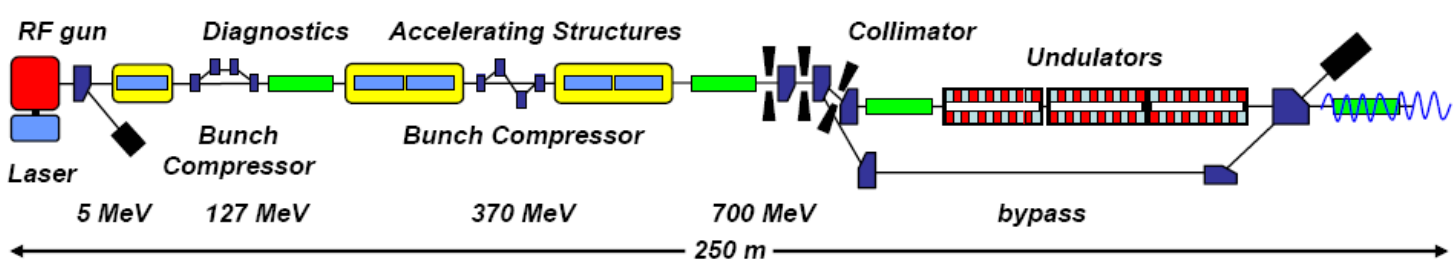

Figure 8. Schematic layout of the second stage of expansion of the TESLA Test Facility, called TTF2. The total length is approximately $250 \mathrm{~m}$, the electron energy $\sim 700 \mathrm{MeV}$.

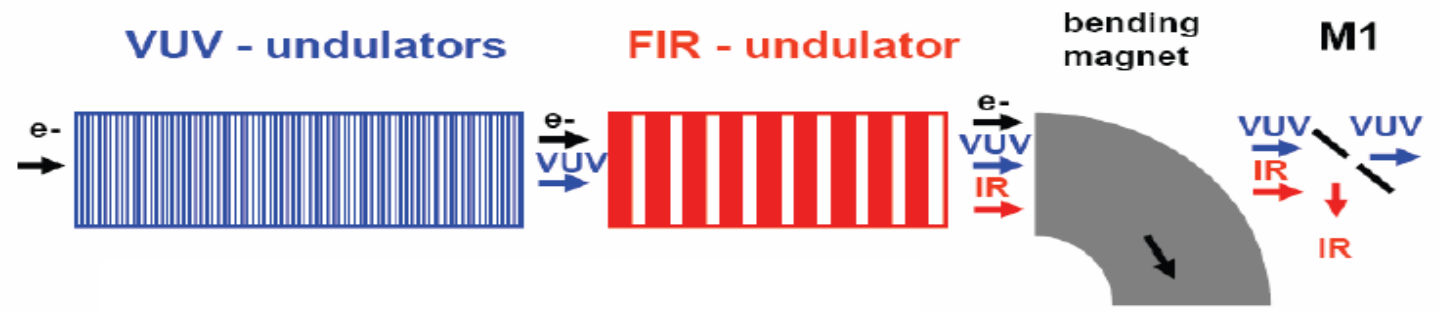

Figure 9. Arrangement of the FIR and VUV undulators in the FLASH accelerator tunnel. The FIR undulator uses the spent electron beam from the VUV undulators. The rather large IR beam is coupled out by a first mirror M1 with a hole in the centre to let pass the much narrower VUV beam [10]. 


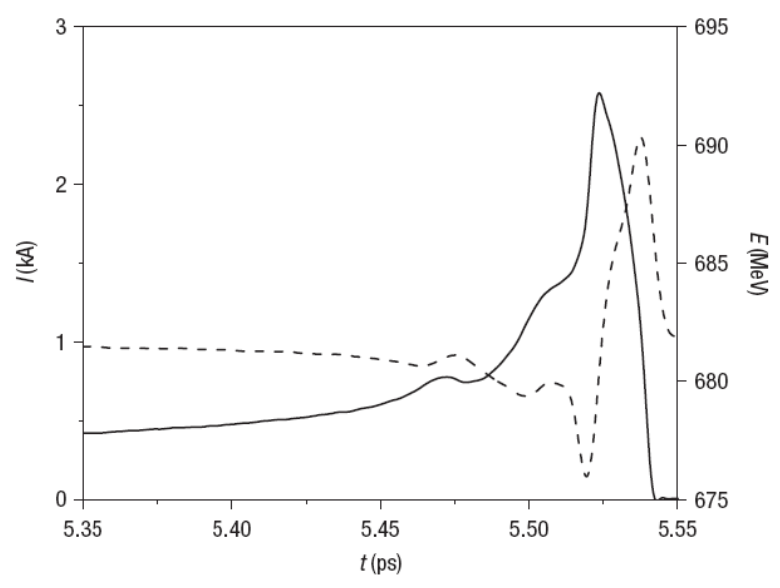

Figure 10. Result of a simulation of the current (solid line) and the mean energy (dashed line) distribution along the electron bunch at the entrance of the undulator. The bunch head is located at the right-hand side of the figure [11].

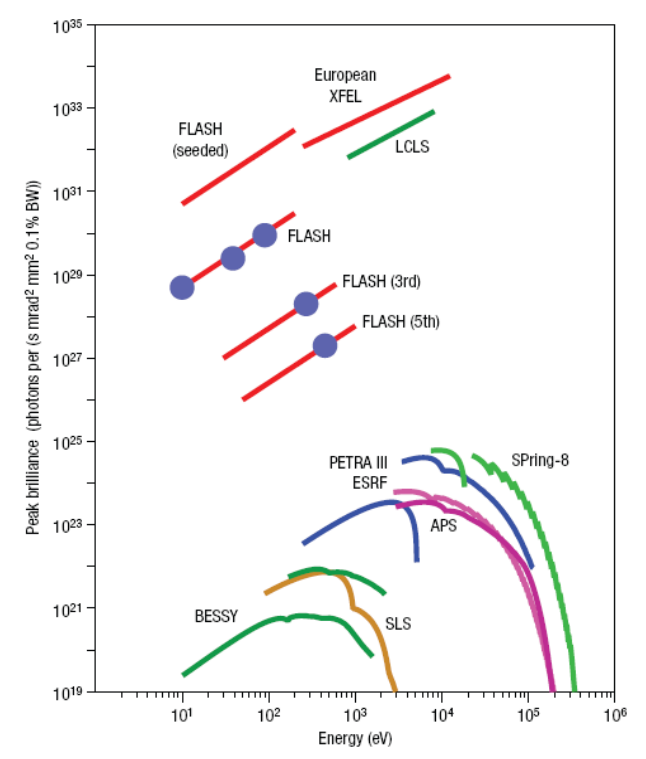

Figure 11. Spectral peak brilliance calculated for FLASH at DESY, the Linac Coherent Light Source (LCLS) at SLAC in Stanford, and the European XFEL Facility in Hamburg in comparison with the performance of $3^{\text {rd }}$ generation synchrotron radiation facilities [9]. Blue spots show the experimental performance of FLASH at the fundamental at $13.7 \mathrm{~nm}$, and at $3^{\text {rd }}$ and $5^{\text {th }}$ harmonics. The curve FLASH (seeded) refers to the proposal for self-seeding of FLASH [6].

\section{First single-shot coherent imaging experiment at FLASH}

One of the most attractive science drivers for X-ray free-electron lasers is the single-shot coherent imaging of nano-scale objects. In order to get enough signal in a single shot image about $10^{12}$ photons focused to a spot of a few microns diameter are needed. On the other hand such high power density will destroy the sample and the question is if one can get the structural information before the target explodes. Model calculations show that in order to achieve this goal the duration of the FEL pulses should be of the order of 10 fsec [14]. 
H. Chapman et al. [15] have performed the proof-of-principle experiment at FLASH using intense $\left(4 \times 10^{13} \mathrm{Wcm}^{-2}\right)$ pulses of $\sim 25 \mathrm{fsec}$ duration which contain $\sim 10^{12}$ photons at $32 \mathrm{~nm}$ wave-

length. The experimental setup is shown in figure 12. The graded multilayer mirror also acts as a filter for the $32 \mathrm{~nm}$ FEL radiation and thus improves the signal to noise ratio on the CCD detector. The single-shot scattering picture is shown in figure 13 together with the reconstructed image. The agreement with the TEM picture of the target shown in the inset of figure 12 is excellent. After transmission of the FLASH pulse the target heats up to about $60,000 \mathrm{~K}$ on picosecond time scales, as a result the scattering picture taken later with a second FLASH pulse corresponds to essentially a hole in the target. Coherent single shot imaging will be discussed in much more detail in following articles.

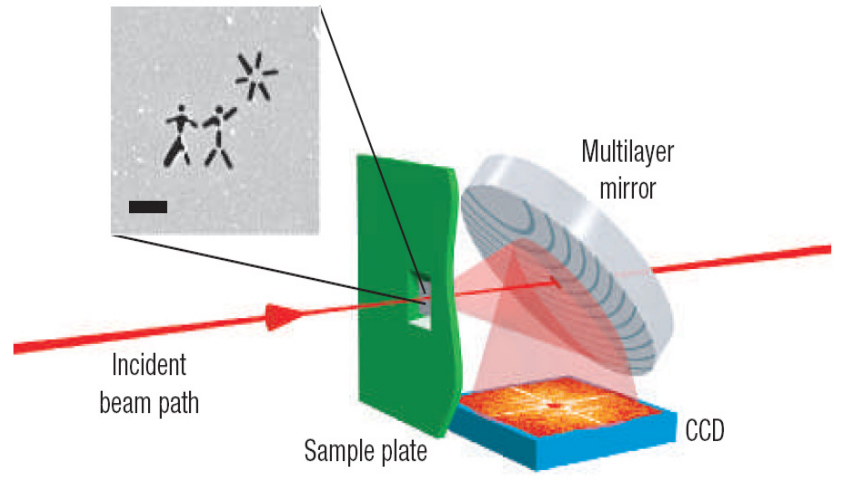

Figure 12. The FEL beam is incident from the left and is focused to a $20 \mu \mathrm{m}$ spot on the sample, which is a 20-nm-thick transmissive silicon nitride membrane with a picture milled through its entire thickness using an FIB (this is enlarged in the inset, and the scale bar indicates $1 \mu \mathrm{m}$ ). The direct beam passes through the sample window and exits the camera through a hole in a graded multilayer planar mirror. The diffracted light from the sample reflects from this mirror onto a CCD detector [15].
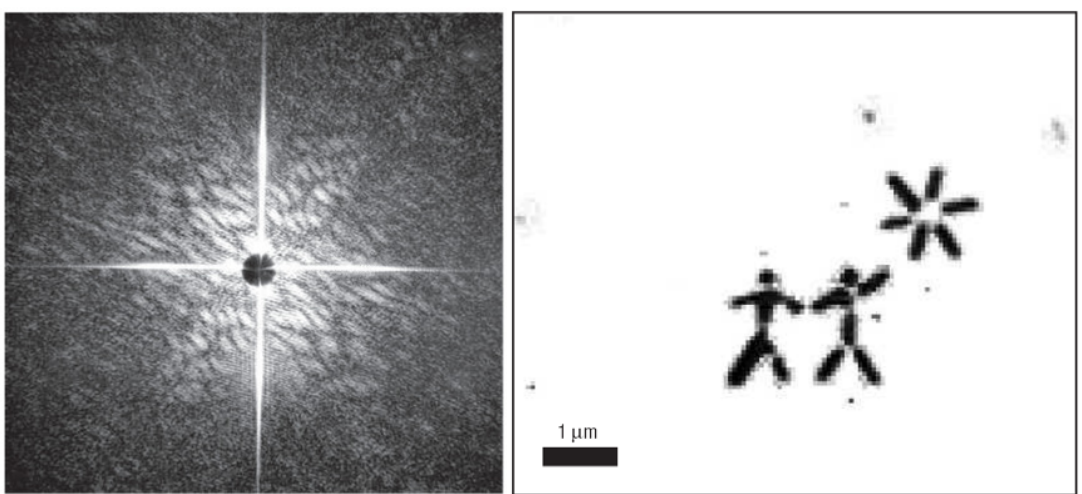

Figure 13. FLASH X-ray coherent diffraction pattern recorded for a single $(4 \pm 2) \times 10^{14}$ $\mathrm{Wcm}^{\cdot 2}, 25 \pm 5 \mathrm{fsec}$ pulse. On the right the reconstructed target is shown which agrees very well with the original shown in the inset of figure 12 [15]

\section{Summary and Outlook}


FLASH is the world's first FEL user facility in the spectral range of the VUV/XUV and many pioneering experiments in a large variety of scientific fields have been performed to date. This includes work on

- Atoms, molecules and clusters

- Matter in extreme conditions, warm dense matter, radiation damage

- Single shot, lens-less imaging of cells and diffraction from nano-scale crystals

- Condensed matter spectroscopy and scattering

- Photon beam diagnostics.

To date more than 96 scientific papers have been published, almost half of them in high impact journals. For an updated list see

http://hasylab.desy.de/facilities/flash/publications/selected_publications/. This list does not include the many publications documenting the enormous progress made in FEL related accelerator science and technology. Here the controlled interaction of strong laser beams with bunches of relativistic electrons is becoming more and more important. More general, as already stated in 1999 by the "Leone Panel", the state-of-the-art light source facility of the future will include a complete marriage of accelerator principles and laser art.

Worldwide three X-ray free-electron lasers are currently in operation, three FELs are under construction, seven more projects are in preparation. Upgrade plans are pursued at DESY and SLAC.

During a shutdown from September 2009 to February 2010 key components of the FLASH injector and many of the RF stations have been exchanged. A $3^{\text {rd }}$ harmonic module was installed which will create a much more homogeneous electron distribution in the bunch. The synchronization and feedback systems, as well as controls in general have been improved. The $1^{\text {st }}$ cryomodule was exchanged and an additional, i.e. the $7^{\text {th }}$ cryomodule was installed, which increases the electron energy to $1.2 \mathrm{GeV}$.

An external seeding option (HHG) has been installed at FLASH and commissioning is expected to start in the fall of 2010. Pulses of $29.6 \mathrm{~nm}$ wavelength and $20 \mathrm{fsec}$ (FWHM) in duration produced via high-harmonics generation are overlaid with the FLASH electron bunches and amplified by passing through $10 \mathrm{~m}$ of undulators. The photon beam is reflected out to an experimental hutch where time-resolved pump-probe experiments will be pursued. The goal is to operate the seeded FEL parasitically to the normal FLASH operation by taking only one bunch out of each bunch train. In addition DESY decided recently to construct an extension of FLASH by adding a second undulator, which will be tunable, and a second experimental hall with 6 experimental stations. FLASH II, as the extension is called, will be a seeded FEL. A model of the facility is shown in figure 12 .

In Japan a VUV FEL has been built as a prototype for the Spring-8 Compact SASE Source (SCSS) aiming for lasing at $0.1 \mathrm{~nm}$ in the fall of 2011. The accelerator test facility started operation for users in May 2008 and provides radiation in the wavelength range from 30 to $61 \mathrm{~nm}$ [16]. In the frame of an international collaboration external seeding by light produced by high-order harmonic generation in a gas was successfully demonstrated [17].

At Stanford in the US the Linac Coherent Light Source (LCLS) demonstrated very rapidly lasing at $0.15 \mathrm{~nm}$ in April 2009, which was again a breakthrough in FEL science and technology [18]. Because of the low emittence of the electron beam only $\sim 80 \mathrm{~m}$ instead of the expected $132 \mathrm{~m}$ of undulator are needed to reach saturation at $0.15 \mathrm{~nm}$. The now spare undulators will be used to build a so called after burner which enhances the intensity of the $2^{\text {nd }}$ harmonic to about the value of the fundamental. Operation for users started in October 2009 providing beam in the spectral range from 0.8 to $2 \mathrm{keV}$ at the AMO station. The experiments benefited largely from the fact that the pulse duration at LCLS could be varied routinely between $\sim 10$ and $300 \mathrm{fsec}$ by changing bunch charge and compression [19]. Plans for further upgrades include a pair of tunable undulators for soft X-rays separated by a magnetic chicane which allow production of a two color beam for jitter free pump-probe experiments on/off resonance. 
In Trieste the single pass free-electron laser FERMI@ELETTRA is under construction. This user facility will cover the wavelength range from $100 \mathrm{~nm}(12 \mathrm{eV})$ to $10 \mathrm{~nm}(124 \mathrm{eV})$ by varying the undulator gap at fixed electron beam energy. It will use a novel concept for external seeding.

The construction of the European XFEL in Hamburg started in January 2009. A linac in superconducting RF technology will serve, in the first stage of expansion, three independent freeelectron lasers covering the wavelength range from 0.1 to $6 \mathrm{~nm}$. This facility combines the extreme peak brilliance characteristic for FELs with a very high average brilliance over the whole wavelength range.

The Paul-Scherrer Institute in Switzerland pursues an XFEL project aiming for wavelengths down to $0.1 \mathrm{~nm}$ using a $6 \mathrm{GeV}$ linac in "warm technology". A $250 \mathrm{MeV}$ prototype linac is currently under construction and the go-ahead for construction of the full facility, called SwissFEL, is expected for 2011.

Further FEL projects are pursued in Frascati, Italy (SPARX), Berkeley, USA (NGLS), Pohang, Korea (PAL-FEL) and in Lund, Sweden (MAX IV-FEL). Other FEL projects are discussed in the UK (NLS), France (ARC-EN-CIEL) and Poland (POLFEL).

All these projects are initiated and supported by a growing user community. The interest for performing experiments at the running FELs is increasing very rapidly. As an example, LCLS issued 3 calls for proposals for experiments targeting the available beamtime from October 2009 to December 2010. 859 scientists (no double counting) from 25 different countries have been involved in 197 proposals. About 1/3 of them got beamtime. This is a rather striking outcome taking into account that LCLS just started operation and that only three of the six LCLS experiments were offered. In average the proposals had 16 authors, a much bigger number then what is typical for proposals submitted to storage ring synchrotron radiation user facilities. These larger user groups are required for experiments at free-electron lasers because a wider range of expertise is needed. In addition the beamtime allocated for a given experiment is short, typically 5 twelve hour shifts at LCLS. As a consequence experiments have to be very well prepared and tested before start of beamtime, loss of beamtime due to technical problems during the run must be minimized.

Because beamtime at X-ray free-electron laser user facilities will always be scarce it is difficult for individual research groups to successfully run a program which largely relies on FEL beamtime. Therefore most groups combine their FEL activities with research in their home laboratories using e.g. optical lasers, or by regular submission of proposals to synchrotron radiation facilities, or both. Some groups go beyond that and form larger collaborations with a high probability that at least one of the member groups has beamtime at any of the FEL facilities available worldwide. These collaborations should have regular meetings where progress is discussed involving actively $\mathrm{PhD}$ students and postdoctoral fellows. At these meetings the scientific goals and the preparation of new experiments could be discussed, e.g. reports on detailed Monte Carlo type simulations of the planed experiments could be presented. A culture which makes the collaboration effective and successful on one hand, and keeps the identity and the contributions of the different groups and people visible, on the other hand, needs to be developed. Then it should also be possible to analyze data jointly within the collaboration and to publish the results together. This approach works in particle and nuclear physics and should be possible to develop on a much smaller scale in the photon science community interested in FEL work

Finally, it is a great pleasure to realize that the FEL user community is very grateful to the accelerator scientists for providing such outstanding research opportunities. Thanks are also due to the funding agencies in several countries which decided to support X-ray free-electron laser work in an early stage when larger parts of the photon science community were still rather reserved with respect to FEL facilities. 


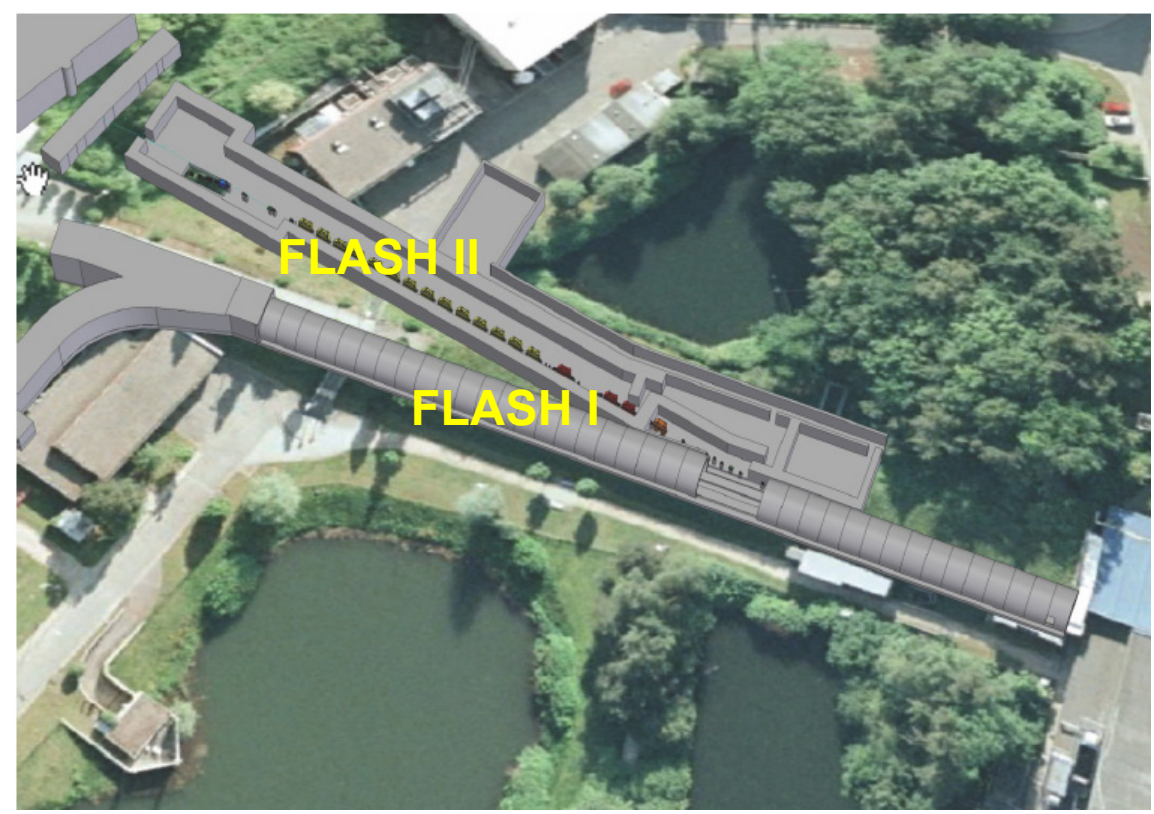

Figure 12. Model of the two branches of the expanded FLASH facility.

\section{References}

[1] Kondratenko A M and Saldin E L 1980 Particle Accelerators 10207

[2] Bonifacio R, Pellegrini C and Narducci L M 1984 Opt. Communications 50373

[3] Brinkmann R, Materlik G, Rossbach J and Wagner A (eds) 1997 "Conceptual Design of 500 $\mathrm{GeV}^{+} \mathrm{e}^{-}$Linear Collider with Integrated X-ray Facility” DESY 1997-048, ECFA 1997-182

[4] Weise H 2002 Proceedings of LINAC2002, Gyeongju, Korea 577-581

[5] Rossbach J 2002 Proceedings of LINAC2002, Gyeongju, Korea 582-586

[6] Feldhaus J, Saldin E L, Schneider J R, Schneidmiller E A and Yurkov M V 1997 Opt. Communications 140 341-352

[7] Wabnitz H et al Nature 420 482-485

[8] Ayvazyan V et al 2006 Eur. Phys. J. D 37 297-303

[9] Ackermann W et al 2007 Nature Photonics $1336-342$

[10] Gensch M et al 2008 Infrared Physics \& Technology 51 423-425

[11] Frühling U et al 2009 Nature Photonics 3 523-528

[12] Mitzner R et al 2008 Optics Express 16 19909-19919

[13] Pietzsch A et al 2008 New Journal of Physics 10 033004/1-10

[14] Neutze R, Wouts R, van der Spoerl D, Weckert E and Hajdu J 2000 Nature 406 752-757

[15] Chapman H N et al 2006 Nature Physics 2 839-843

[16] Shintake T et al 2008 Nature Photonics 2 555-559

[17] Lambert G et al 2008 Nature Physics 4 296-300 
[18] Emma P (for the LCLS Commissioning Team) 2009 Proceedings of PAC2009, Vancouver, Canada

[19] Ding Y et al 2009 Phys. Rev. Lett. 102 254801/1-4 